\title{
Lower Volga Valley Phytosociological Database
}

\author{
Valentin Golub, Alexey Sorokin, Kseniya Starichkova, Lyudmila Nikolaychuk, Viktoria \\ Bondareva \& Tatyana Ivakhnova
}

\begin{abstract}
The structure and content of the vegetation database of the Lower Volga Valley are presented (Lower Volga Valley Phytosociological Database, GIVD ID EU-RU-002). The lower part of the Volga valley comprises two parts, the Volga-Akhtuba flood-plain and the Volga delta. Traversing the arid Caspian lowland, the valley of the Volga lower section is remarkable for a broad variety of vegetation. All available phytosociological relevés of different classes have been collected (Charetea, Lemnetea, Ruppietea maritimae, Potametea, Phragmito-Magno-Caricetea, Isoëto-Nano-Juncetea, Crypsidetea aculeatae, Artemisietea lerchianae, Artemisietea tchernievianae, Oryzetea sativae, Chenopodietea, Secaletea, Glycyrrhizetea glabrae, Molinio-Arrhenatheretea, TheroSalicornietea strictae, Salicornietea fruticosae, Nerio-Tamaricetea, Salicetea purpureae, Querco-Fagetea) and stored in a TURBOVEG 2.88 database. Currently, 13,000 relevés are available in the database. The data are mainly used for plant cover classification and studying vegetation dynamics.
\end{abstract}

Keywords: ecoinformatics; Volga-Akhtuba flood-plain; Volga delta.

\section{Lower Volga Valley Phytosociological Database}

Scope: All available relevés of different classes (Charetea, Lemnetea, Ruppietea maritimae, Potametea, Phragmito-Magno-Caricetea, IsoëtoNano-Juncetea, Crypsidetea aculeatae, Artemisietea lerchianae, Artemisietea tchernievianae, Oryzetea sativae, Chenopodietea, Secaletea, Glycyrrhizetea glabrae, Molinio-Arrhenatheretea, Thero-Salicornietea strictae, Salicornietea fruticosae, Nerio-Tamaricetea, Salicetea purpureae, Querco-Fagetea) have been collected from the Volga-Akhtuba flood-plain and the Volga delta.

Status: completed and continuing

Period: 1928-2011

Database manager(s): Valentin Golub (vbgolub2000@mail.ru); Alexey Sorokin (an-sorokin@yandex.ru); Kseniya Starichkova (kseniyastarichkova@yandex.ru)

Owner: Laboratory of Phytocenology, Institute of Ecology of the Volga River Basin of Russian Academy of Sciences

Web address: http://www.phytosociology.narod.ru/

Availability: according to a specific agreement

Online upload: no

Online search: no

Database format(s): TURBOVEG

Export format(s): TURBOVEG

Publication: Golub, V. B., Sorokin, A. N., Ivakhnova, T. L., Starichkova, K. A., Nikolaychuk, L. F., Bondareva, V. V. (2009): Geobotanicheskaja baza dannykh doliny Nizhney Volgi [Lower Volga Valley Phytosociological Database]. - Izvestiya Samarskogo nauchnogo tzentra RAN. Vol. 11, N 1(4): 577-582, Samara (in Russian).

Plot type(s): normal plots; time series Plot-size range: $0.15-2500 \mathrm{~m}^{2}$

Non-overlapping plots: 13,280

Total plot observations: 13,280

Estimate of existing plots: 15,000

Number of sources: [NA]

Countries: KZ: $1.0 \%$; RU: $99.0 \%$

Forest: [NA] — Non-forest: [NA]

Guilds: all vascular plants: $100 \%$; bryophytes (terricolous or aquatic): $5 \%$; lichens (terricolous or aquatic): $2 \%$; algae (terricolous or aquatic): $3 \%$; non-terricolous taxa (epiphytic, saxicolous, lignicolous): $1 \%$

Environmental data: altitude: $8 \%$; slope aspect: $1 \%$; slope inclination: $1 \%$; microrelief: $34 \%$; other soil attributes: $20 \%$; land use categories: $3 \%$

Performance measure(s): cover: $100 \%$; measurements like diameter or height of trees: $1 \%$

Geographic localisation: GPS coordinates (precision $25 \mathrm{~m}$ or less): $15 \%$; point coordinates less precise than GPS, up to $1 \mathrm{~km}: 85 \%$

Sampling periods: 1920-1929: 2.0\%; 1930-1939: 2.0\%; 1950-1959: 9.0\%; 1960-1969: 3.0\%; 1970-1979: 13.0\%; 1980-1989: 33.0\%; 1990-1999: 13.0\%; 2000-2009: 5.0\%; 2010-2019: 13.0\%

Information as of 2012-07-25; further details and future updates available from http://www.givd.info/ID/EU-RU-002

Valentin Golub (vbgolub2000@mail.ru), Kseniya Starichkova (kseniya-starichkova@yandex.ru), Lyudmila Nikolaychuk (vbgolub2000@mail.ru), Viktoria Bondareva (victoria_bondareva@rambler.ru), Tatyana Ivakhnova (aquarius_tlt@mail.ru) Laboratory of Phytosociology, Institute of Ecology of the Volga River Basin of Russian Academy of Sciences, Komzina str. 10, 445003 Togliatti, RUSSIA

Alexey Sorokin* (an-sorokin@yandex.ru)

Laboratory Phytocenology, Institute of Ecology of the Volga River Basin of Russian Academy of Sciences, Komzina str. 10, 445003

Togliatti, RUSSIA

*Corresponding author 